\title{
Efficacy and tolerability of low-dose oral prolonged-release oxycodone/naloxone for chronic nononcological pain in older patients
}

This article was published in the following Dove Press journal:

Clinical Interventions in Aging

16 December 2014

Number of times this article has been viewed

\section{Fabio Guerriero ${ }^{1,2}$ \\ Carmelo Sgarlata ${ }^{2}$ \\ Claudio Marcassa ${ }^{3}$ \\ Giovanni Ricevuti',2 \\ Marco Rollone'}

'Azienda di Servizi alla Persona of Pavia, Istituto di Cura Santa Margherita, Pavia, Italy; ${ }^{2}$ Department of Internal Medicine and Medical Therapy, University of Pavia, Pavia, Italy; ${ }^{3}$ Fondazione Salvatore Maugeri IRCCS, Veruno, Novara, Italy

Video abstract

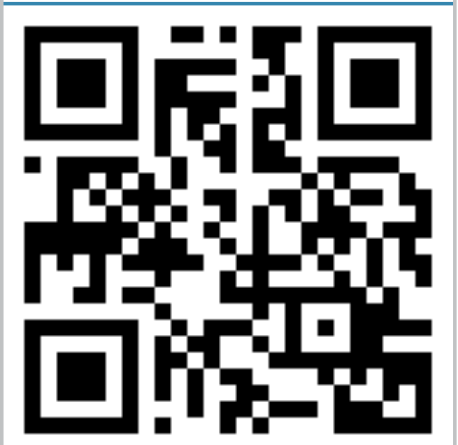

Point your SmartPhone at the code above. If you have a QR code reader the video abstract will appear. Or use:

http://dvpr.es/IxTEAWs
Correspondence: Fabio Guerriero Istituto di Cura S Margherita, via Emilia, 12, 27100 Pavia, Italy Tel +390382 38I 772

$\mathrm{Fax}+390382381218$

Email fabio_guerriero@yahoo.it
Purpose: Chronic pain is highly prevalent in older adults. Increasing evidence indicates strong opioids as a valid option for chronic pain management in geriatrics. The aim of this study was to evaluate efficacy and safety of low-dose oral prolonged-release oxycodone-naloxone (OXN-PR) in patients aged $\geq 70$ years.

Methods: This open-label prospective study assessed older patients naïve to strong opioids presenting with moderate-to-severe chronic pain. Patients were prescribed OXN-PR at an initial dose of $10 / 5 \mathrm{mg} /$ day for 28 days. In case of insufficient analgesia, the initial daily dose could be increased gradually. The primary efficacy measure was change in pain intensity from baseline, assessed by a ten-point Numeric Rating Scale (NRS) at day 28 (T28). Changes in cognitive state, daily functioning, quality of life, constipation, and other adverse events were assessed.

Results: Of 53 patients enrolled (mean 81.7 \pm 6.2 years [range 70-92 years]), 52 (98.1\%) completed the 28 -day observation. At $\mathrm{T} 28$, the primary end point ( $\geq 30 \%$ reduction in mean pain from baseline in the absence of bowel function deterioration) was achieved in 38 patients (71.7\%). OXN-PR significantly relieved pain (NRS score $-3.26 ; P<0.0001$ ), as well as daily need for rescue paracetamol (from $86.8 \%$ at baseline to $40.4 \%$ at T28; $P<0.001$ ), and reduced impact of pain on daily activities (Brief Pain Inventory Short Form from $6.2 \pm 1.5$ to $3.4 \pm 2.1$; $P<0.0001)$. OXN-PR was also associated with significant improvement in daily functioning (Barthel Index from $53.3 \pm 14.1$ to $61.3 \pm 14.3 ; P<0.01$ ). No changes were observed in cognitive status and bowel function. OXN-PR was well tolerated; only one patient (1.9\%) prematurely withdrew from treatment, due to drowsiness.

Conclusion: Findings from this open-label prospective study suggest that low-dose OXN-PR may be effective and well tolerated for treatment of moderate-to-severe chronic pain in older patients. Besides its effectiveness, these data indicate that low-dose OXN-PR may be considered a safe analgesic option in this fragile population and warrants further investigation in randomized controlled studies.

Keywords: chronic pain, elderly, opioid, oxycodone, constipation

\section{Introduction}

Chronic pain is common in older people, affecting about $60 \%$ of people aged $>65$ years and functionally impairing $45 \%-80 \%$ of older people in nursing homes. ${ }^{1}$ Pain of musculoskeletal origin increases with age. In particular, pain associated with degenerative joint disease (eg, osteoarthritis) shows an exponential increase until at least 90 years of age. ${ }^{2}$ In fact, chronic pain is predominantly nociceptive in older adults, although neuropathic or mixed pain is also common. ${ }^{3}$ Chronic pain gives rise to an increase in costs and utilization of health care resources and, particularly in older people, has a significant impact on the quality of life, leading to a decrease in social 
activities and an increase in sleep disorders, depression, anxiety, cognitive decline, malnutrition, and risk of falls. ${ }^{2,4}$

Opioid analgesics can be an effective therapy for chronic pain and are also recommended for older patients with moderate-to-severe pain or for pain that impairs functioning and quality of life, and should be administered as part of a comprehensive pain management strategy. ${ }^{4}$ Major barriers to the use of opioids in older patients are the fear of opioidrelated adverse events (AEs) and concerns about the development of drug dependence. ${ }^{4-6}$ The uncertainty surrounding the potential causal role of opioids in cognitive impairment may also contribute to the reluctance to prescribe opioids. The most frequently reported opioid-induced AEs are constipation, sedation, respiratory depression, nausea/vomiting, and delirium. ${ }^{3}$ Unlike most AEs that resolve with prolonged opioid use due to the development of tolerance, constipation is persistent and is a frequent cause of dose reduction or treatment discontinuation..$^{7-9}$ In elderly patients, constipation can be an even more relevant problem, as predisposition to bowel dysfunction increases with age. ${ }^{4,10}$

To prevent or reduce opioid-induced bowel dysfunction, a novel, oral, prolonged-release formulation of the combination of oxycodone, a strong opioid, and naloxone, an opioid receptor antagonist, was recently developed. ${ }^{11}$ The rationale for the development of the combination oxycodone-naloxone (OXN-PR) was based on the fact that oral naloxone, due to its very low systemic bioavailability, predominantly antagonizes opioid receptors in the gastrointestinal tract, thereby preventing bowel dysfunction induced by oxycodone, without affecting the central, analgesic effects of oxycodone. ${ }^{6,12,13}$ The oral prolonged-release OXN-PR combination was shown in a number of controlled randomized trials to provide effective analgesia to patients with severe chronic pain and to significantly reduce the incidence of opioid-induced constipation, with an improvement in compliance and quality of life. ${ }^{14-16}$

The objective of this prospective observational study was to investigate the analgesic efficacy and tolerability of low doses of OXN-PR in older patients ( $\geq 70$ years) naïve to strong opioids. The effects of OXN-PR on functional and cognitive status, mood, and quality of life were also analyzed.

\section{Materials and methods Study design and patients}

This 4-week, single-center, prospective observational study was performed at Santa Margherita Institute, Pavia, Italy - a center specialized in physical rehabilitation of older people.
The study was approved by the local Institutional Review Board. All patients provided written informed consent for the collection and analysis of their anonymous demographic and clinical data. Consecutive outpatients seen in our Geriatric Unit and receiving a new prescription for OXN-PR were considered for this observation. To be eligible for inclusion, patients had to meet the following criteria: age $\geq 70$ years; suffering from chronic pain (duration $\geq 3$ months) of various nononcologic etiologies and of moderate-to-severe intensity (Numeric Rating Scale [NRS] score $\geq 4$ ), despite analgesic treatment (nonsteroidal anti-inflammatory drugs [NSAIDs], paracetamol alone or in combination with low doses of codeine or tramadol); naïve to World Health Organization (WHO) step III opioid analgesics; and absence of contraindications to oxycodone use.

Patients with one of the following were excluded: inability to take oral medication; symptomatic cerebral metastases; known psychiatric disease; cognitive impairment or dementia not allowing appropriate pain assessment (score $<18$ at Mini-Mental State Examination [MMSE; range 0-30], with lower scores indicating more severe cognitive impairment) $;{ }^{17}$ severe chronic obstructive pulmonary disease or other respiratory disease with hypoxemia and/or hypercapnia; paralytic ileus; moderate or severe liver insufficiency (total bilirubin $\geq 1.5 \mathrm{mg} / \mathrm{dL}$ ); moderate or severe renal insufficiency (glomerular filtration rate $<30 \mathrm{~mL} /$ minute according to Cockcroft-Gault formula); or hypersensitivity to the active substances or to any of the excipients of the study medication.

Patients were visited by specialized clinicians trained in the management of pain at the beginning of the observation when OXN-PR was initiated (T0) and after 7 days (T7), 14 days (T14), and 28 days (T28) of treatment. Every $\mathrm{AE}$ or other inconvenience related to the drug was also investigated, and the daily OXN-PR dose was adjusted accordingly.

\section{Treatment}

Opioid therapy was initiated after the T0 visit, according to current prescription recommendations in older patients, ${ }^{4,10}$ and previous analgesics were discontinued. The oral OXN-PR combination (Targin ${ }^{\circledR}$, Mundipharma Pharmaceuticals, Milan, Italy) was started at a low dose $(5 / 2.5 \mathrm{mg}$ twice daily), with the first tablet administered at $8 \mathrm{pm}$.

After 7 days, in case of insufficient analgesia, the daily dose of OXN-PR could be increased to $15 / 7.5 \mathrm{mg}$, and finally up to $20 / 10 \mathrm{mg}$ after 14 days (the maximum daily dose of OXN-PR that was expected in the present study). 
As this was an exploratory investigation in a fragile population suffering from pain lasting longer than 3 months, with limited data on OXN safety/tolerability and titration in elderly patients, OXN-PR was started at a low dose, with a 7-day interval for the first titration. To control pain during this time and at any time during the study, all patients were instructed to take a dose of rescue on-demand paracetamol $500 \mathrm{mg}$ when they did not achieve satisfactory pain control (defined as a pain intensity scale ["pain right now"] score $\geq 5$ ). In case of rescue medication dosing more than twice in 1 day, patients were instructed to contact our center in advance of their next follow-up visit.

Adjuvant drugs and other medications used for the treatment of any other underlying medical condition, if taken, were continued with no change in their dosage.

\section{Assessments}

Demographic information and details of clinical history, location and cause of pain, and treatments for pain and constipation were recorded at entry visit. Pain intensity the primary efficacy measure - was assessed on a verbally administered zero to ten-point NRS (where $0=$ no pain, $1-3=$ mild pain, $4-6=$ moderate pain, and $7-10=$ severe pain) at rest, during movement, during the day, and at night-time, and the corresponding NRS values were computed as a mean of the last 7 days.

T0 evaluation also included 1) the cognitive status assessment using MMSE; 2) daily functioning, evaluated by the Barthel Index (the maximum score achievable, 100, indicates that the patient can perform all activities independently); ${ }^{18}$ 3) interference of pain in the patient's life, as reported by the patient and evaluated by the seven-item composite pain interference included in the Brief Pain Inventory Short Form (BPI-SF; general activity, walking ability, normal work, mood, enjoyment of life, sleep, and relations with other people) with an eleven-point NRS for each item, ranging from $0=$ no interference to $10=$ complete interference: ${ }^{19}$ an Italian version of the BPI has been previously validated; ${ }^{20}$ and 4 ) constipation, either spontaneous or opioid induced, and bowel function in the last 7 days, evaluated by the Bowel Function Index (BFI) according to Rentz et al. ${ }^{21}$ The BFI is a measure of general bowel function validated as a reproducible tool that detects clinically meaningful changes in constipation, with scores ranging from 0 (free from symptoms) to 100 (most severe symptoms). In patients with chronic pain, normal bowel function is defined as a BFI value of $\leq 29$, and a BFI value change of $\geq 12$ points represents a clinically meaningful change in constipation severity. ${ }^{22}$
The effectiveness variables were also assessed at T7, T14, and T28 time points, and the daily functioning assessment was repeated at $\mathrm{T} 28$.

Safety evaluations were performed at baseline and at $\mathrm{T} 7, \mathrm{~T} 14$, and $\mathrm{T} 28$ time points by recording presence and severity (on a verbally administered $0-10$ NRS, where $0=$ absence and $10=$ extreme severity) of symptoms commonly related to opioid treatment (nausea, mouth dryness, dizziness, drowsiness, tremor, and pruritus) and any other treatment-related $\mathrm{AE}$ that occurred or worsened in intensity and/or frequency after the first intake of OXN-PR. The potential correlation between the $\mathrm{AE}$ and the treatment with OXN-PR was judged by the pain physician, considering AEs of any intensity.

\section{Statistical analysis}

The primary end point of the trial - the rate of favorable response to the new oral analgesic combination - was defined as the proportion of patients who achieved a $\geq 30 \%$ reduction in pain intensity score from baseline to 28 days of treatment with the OXN-PR combination, in the absence of further bowel function impairment (ie, BFI score increase $<12$ points).

To determine the size of the sample to be included in the observation, it was estimated that clinically relevant observations could be made on a population composed of at least $70 \%$ of responders; given the low dosages of OXN-PR planned for this observation, the lower limit of the observed patient proportion was set at $50 \%$. With a power of $80 \%$ and a significance level of 0.05 , a minimum of 49 patients was needed. In order to allow for dropouts and incomplete data $(\cong 10 \%)$, we decided to include at least 55 patients.

In the event of missing values, the last observation carried forward approach was used to impute missing data. Normality distributions of continuous variables were assessed by the Shapiro-Wilk test. Data are reported as mean \pm standard deviation or median and range. The significance of differences between pairs of continuous variables was evaluated by the Student's $t$-test or the Wilcoxon test, as appropriate. Change in continuous variables over time were evaluated by analysis of variance (with a post hoc Bonferroni's correction to adjust for multiple comparisons) or by Kruskal-Wallis analysis, as appropriate. Changes over time in categorical variables were compared using the Cochran's $Q$ test. A $P$-value $<0.05$ was considered significant. Analysis was performed with STATISTICA version 10 (StatSoft, Tulsa, OK, USA). 


\section{Results}

\section{Baseline clinical characteristics}

Between January and September 2013, 147 patients aged $\geq 70$ years suffering from chronic pain (duration $\geq 3$ months) of various nononcologic etiologies and of moderateto-severe intensity (NRS score $\geq 4$ ) despite analgesic treatment were considered for this study; 126 patients were naïve to WHO step III opioid analgesics. We excluded 38 patients due to concomitant illness, oxycodone contraindications, and other physical limitations; 32 others were not included due to logistic impediments to completing the planned 4-week follow-up or because they did not provide informed consent. Among the remaining 56, three subjects were excluded due to cognitive impairment according to their MMSE score $(<18)$, while 53 patients fulfilled the selection criteria and were included in the final analysis (36.0\% of the screened population). The study flow chart is shown in Figure 1.

The demographic and baseline clinical characteristics of the 53 enrolled patients are shown in Table 1. Of note, patients' median age was 83 years, including 17 subjects who were aged $\geq 85$ years. At entry visit, the enrolled population was suffering from pain with a median NRS score of 6 , and $34(64.2 \%)$ patients were complaining of severe pain (ie, NRS score $>6$ ) at baseline. As expected for an older population, pain was nociceptive in almost all patients $(\mathrm{n}=52,98.1 \%)$, mostly caused by osteoarthritis $(62.2 \%)$; in 13 subjects $(24.5 \%)$, pain was due to sequelae of femur or vertebral fractures. The pain lasted more than 3 and 6 months in $35(66.0 \%)$ and $13(24.5 \%)$ patients, respectively. At baseline, $86.8 \%$ of patients required daily rescue paracetamol and none received adjunctive therapy (ie, steroids, antidepressants, or anticonvulsants).

According to the selection criteria, mean baseline MMSE score (25.8 \pm 2.6$)$ suggested the absence of overt cognitive impairment in the evaluated population.

\section{OXN-PR and adjunctive therapy during the study}

All patients started opioid treatment with low daily doses of OXN-PR (10/5 mg twice daily). During the first week, only one patient $(1.9 \%)$ discontinued the new treatment, due to unpleasant drowsiness. Of the remaining 52 patients, the majority kept their OXN-PR dosages unchanged throughout the observation period, as illustrated in Figure 2; the mean daily dose of oxycodone-equivalent at study end was $14.1 \pm 5.2 \mathrm{mg}$ (median 10 [range 5-20 mg]).

None of the patients received adjunctive therapy (ie, steroids, anticonvulsants, or antidepressants) before enrollment or at any time during this observation.

For all the considered variables, we had no missing data.

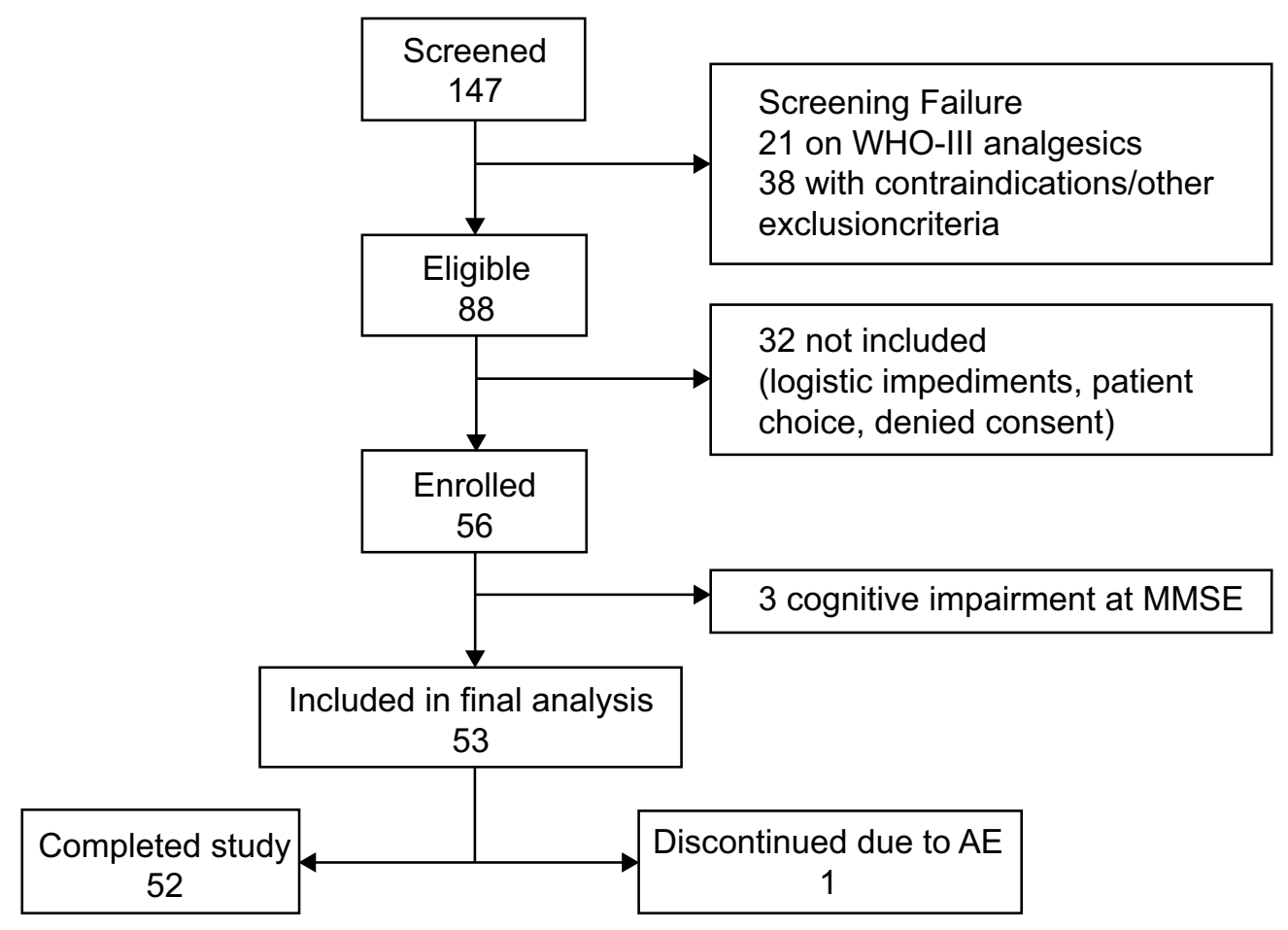

Figure I Patient disposition. 
Table I Demographic and baseline characteristics of the study population

\begin{tabular}{ll}
\hline Parameter & Value \\
\hline $\mathrm{N}$ & 53 \\
Age, years (median; range) & $81.8 \pm 5.7(83 ; 70-94)$ \\
Patients >80 years, $\mathrm{n}(\%)$ & $37(69.8 \%)$ \\
Females/males, $\mathrm{n}(\%)$ & $44 / 9(83.0 / 17.0)$ \\
Pain intensity, mean NRS score* & $6.7 \pm 1.2$ \\
Pain intensity at rest, NRS score & $5.9 \pm 1.9$ \\
Pain intensity on movement, NRS score & $8.0 \pm 0.9$ \\
Daytime pain intensity, NRS score & $7.5 \pm 1.0$ \\
Nocturnal pain intensity, NRS score & $5.4 \pm 2.1$ \\
Previous analgesic treatment, $\mathrm{n}(\%)$ & \\
Step I WHO drugs & \\
NSAIDs & $6(11.3)$ \\
Paracetamol & $36(67.9)$ \\
Daily dosage, mg (mean) & $\mathrm{I}, 796 \pm 857 \mathrm{mg}$ \\
Step II WHO drugs & $20(37.7)$ \\
Codeine ${ }^{\dagger}$ & $3(5.6)$ \\
Daily dosage, mg (mean) & $60 \pm 0$ \\
Tramadol & $17(32.0)$ \\
Daily dosage, mg (mean) & $129.7 \pm 52.6$ \\
Rescue analgesics, $\mathrm{n}$ (\%) & $46(86.8)$ \\
Cognitive state, MMSE score (median) & $25.8 \pm 2.6(26)$ \\
Daily functioning, Barthel score (median) & $53.3 \pm I 4.1(55)$ \\
Bowel function, BFI score (median) & $36.4 \pm 20.0(40)$ \\
\hline Notes Al vas are &
\end{tabular}

Notes: All values are expressed as numbers (percent) or mean $( \pm$ standard deviation) if not otherwise reported. The sum of percentages may not equal 100 , due to rounding. *Expressed as mean of assessments for all four items (pain at rest, on movement, daytime, and nocturnal). ${ }^{\dagger}$ At the time of this observation, available only in combination with paracetamol (30/500 mg each) without temporal limitations (currently $<72$ hours).

Abbreviations: BFI, Bowel Function Index; MMSE, Mini-Mental State Examination; NRS, Numeric Rating Scale; NSAIDs, nonsteroidal anti-inflammatory drugs; WHO, World Health Organization.

\section{OXN-PR efficacy}

Despite the low OXN-PR doses used, the new treatment was associated with a substantial decrease in reported pain intensity. At T28, the primary end point of our investigation, a reduction of mean daily pain intensity $\geq 30 \%$ of the corresponding baseline values in the absence of bowel function deterioration was achieved in 38 patients $(71.7 \%)$; mean pain intensity decrease at T28 was $-47.5 \% \pm 28.1 \%$ (median $-50 \%$ [range $+4.8 \%,-100 \%]$ ).

Individual changes of pain intensity from baseline to T28 are reported as a "waterfall" graph in Figure 3. Pain assessment showed a constant decrease in every measurement (ie, at rest and during movement, during the daytime and night-time) (Table 2); overall mean NRS score markedly decreased from baseline to T28 (from $6.7 \pm 1.2$ to $3.4 \pm 1.7$, $P<0.0001$ ).

Although at T28 there were 15 patients $(28.3 \%)$ who did not achieve a reduction in mean daily pain $\geq 30 \%$ of

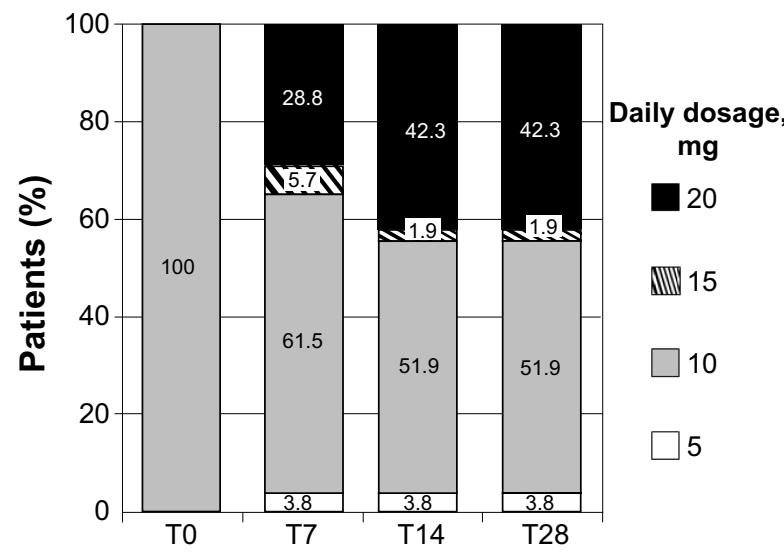

Figure 2 Distribution of prolonged-release oxycodone-naloxone (OXN-PR) daily dosages throughout the observation (expressed in oxycodone-equivalents). The $15 \mathrm{mg}$ daily dosage was two $5 / 2.5 \mathrm{mg}$ tablets in the morning and one in the evening, or vice versa, according to individual need and pain characteristics (three patients at day 7 [T7], one at day 14 [TI4], and one at day 28 [T28]). There were two women (aged 75 and 80 years) who, after a few days of the new treatment, reduced their OXN-PR daily doses to only one $5 / 2.5 \mathrm{mg}$ tablet in the morning because of drowsiness; in the following weeks, both increased their OXN-PR daily dose to $20 / 10 \mathrm{mg}$ without major side effects. Two other women with severe knee pain due to osteoarthritis at the last follow-up visit had decreased their daily OXN-PR to one $5 / 2.5 \mathrm{mg}$ tablet in the morning, nevertheless with satisfactory pain control.

their corresponding baseline values, only four patients (7\%) were still complaining of severe (mean NRS $>6$ ) pain, while 18 patients $(34.0 \%)$ achieved complete or near complete benefit (mean NRS $=0-2$ ) (Figure 4).

Daily need for rescue paracetamol decreased from $86.8 \%$ at baseline to $40.4 \%$ at T28; $(P<0.001)$. No patients contacted the center due to needing to take rescue medication more than twice in 1 day at any point during the study.

Pain-related functional impairment, as reflected by the BPI-SF composite pain interference score in daily life, substantially declined throughout the study (from $6.2 \pm 1.5$ to $3.4 \pm 2.1 ; P<0.0001)$. All seven individual domain scores gradually decreased from baseline to T28 (Figure 5).

After OXN-PR treatment, no substantial changes were observed in cognitive status as assessed by MMSE (from $25.8 \pm 2.6$ at baseline to $26.0 \pm 2.6$ at day 28 ; not significant [NS]), this small increase being of no clinical relevance. On the contrary, there was a slight improvement in the ability to perform daily activities, as shown by a significant increase in the Barthel Index score (from $53.3 \pm 14.1$ to $61.3 \pm 14.3 ; P<0.01$ ).

\section{Tolerability and safety}

Although only 14 (26.4\%) patients complained of constipation at baseline, almost all (96.2\%) had abnormal BFI values (ie, >29), indicating some degree of bowel dysfunction (median BFI value 40). The administration of OXN-PR was associated with a slight and clinically nonsignificant 


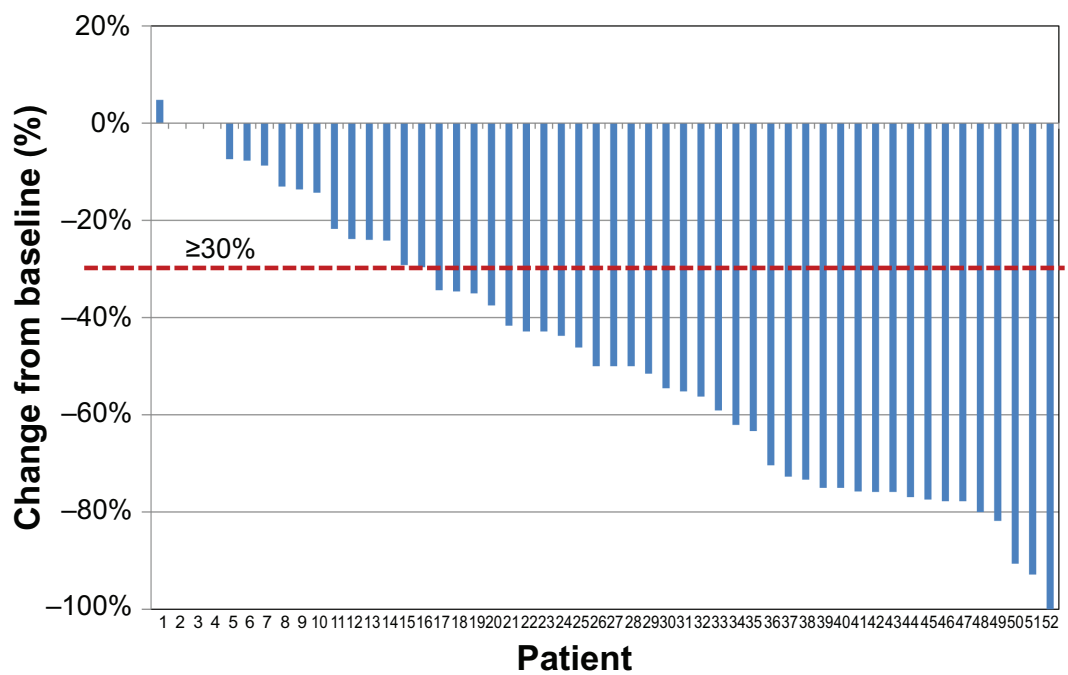

Figure 3 "Waterfall" image of ultimate individual variations in pain severity (last 7 days mean values, Numeric Rating Scale) at the end of observation after 4 weeks of treatment with prolonged-release oxycodone-naloxone among the 52 patients who completed the observation: percentage changes from baseline values are reported. Below the dashed line indicates analgesic efficacy ( $\geq 30 \%$ decrease in mean Numeric Rating Scale).

decrease $(-4.0 \pm 7.9 ; P=\mathrm{NS})$ in BFI values throughout the study (from 36.4 \pm 20.0 at baseline to $33.1 \pm 18.8$ at T28; $P=\mathrm{NS}$ ) (Figure 6); of note, none of the patients had an increase in BFI values from baseline. At last follow-up, fewer patients were still complaining of constipation $(n=8,15.4 \%$; odds ratio [OR] 0.51, 95\% confidence interval [CI] 0.19-1.34; $P=\mathrm{NS}$ vs baseline). Laxative consumption did not change significantly during the observation (20/53 [37.7\%] at baseline vs 22/52 [42.3\%] at T28; OR 1.21, 95\% CI 0.55-2.64; $P=\mathrm{NS})$.

Overall, OXN-PR was well tolerated during the 28 days of treatment. Of the 53 patients who started treatment, only one patient (1.9\%) experienced a severe AE (drowsiness) leading to OXN-PR discontinuation within the first week. Figure 7 shows the prevalence and severity of common opioid-related symptoms at different time points of the observation (ie, nausea, tremor, drowsiness, dizziness, itching, and dry mouth). Almost all symptoms were already present at baseline and, as a matter of fact, decreased during the study; only dry mouth worsened slightly over the 4-week observation. Differences in the pattern of AEs throughout the study were not significant, due to the small number of reported AEs. No other side effects were reported.

\section{Discussion}

Pain is often under-recognized in the older population due to multiple concomitant chronic diseases and sensory, cognitive, and psychological problems. Older people may be reluctant to seek medical help for pain, due to the prevailing idea that pain is inevitably associated with aging, but also due to patients' fear of treatments and related side effects. ${ }^{1}$ Surveys on real-life geriatric management of pain show that elderly patients are all too often inappropriately treated. For example, they often experience insufficient pain relief with NSAIDs alone for extended periods before starting opioid analgesics. ${ }^{10,23}$ Opioid use is often unnecessarily postponed for fear of AEs; however, especially in elderly patients at high risk of NSAID-induced AEs such as gastrointestinal bleeding, strong opioids at low dose may, in fact, be a bettertolerated treatment. ${ }^{4}$

Table 2 Change in pain intensity (Numeric Rating Scale score) during the 28-day observation of oxycodone/naloxone prolongedrelease treatment

\begin{tabular}{|c|c|c|c|c|c|}
\hline Parameter & Baseline & Day 7 & Day I4 & Day 28 & $\begin{array}{l}\text { Analysis of } \\
\text { variance } P \text {-value* }\end{array}$ \\
\hline Pain at rest, mean & $5.9 \pm 1.9$ & $4.6 \pm 1.6^{\dagger}$ & $3.9 \pm 1.8$ & $2.9 \pm 1.7^{\#}$ & $<0.0001$ \\
\hline Pain on movement, mean & $8.0 \pm 0.9$ & $6.3 \pm 1.6 \pi$ & $5.3 \pm 1.9^{\#}$ & $4.3 \pm 2.0^{\#}$ & $<0.0001$ \\
\hline Daytime pain, mean & $7.5 \pm 1.0$ & $5.9 \pm 1.7 \pi$ & $5.0 \pm 2.1$ & $3.9 \pm 2.3^{\dagger}$ & $<0.0001$ \\
\hline Nocturnal pain, mean & $5.4 \pm 2.1$ & $4.2 \pm 2.0^{\#}$ & $3.5 \pm 2.1$ & $2.5 \pm 1.9^{\wedge}$ & $<0.0001$ \\
\hline
\end{tabular}

Notes: Included in this analysis were all patients completing the 28 -day treatment $(n=52)$. *Day 28 vs baseline; ${ }^{\wedge} P<0.0$ I vs previous observation; ${ }^{*} P<0.0$ I vs previous observation; ${ }^{\dagger} P<0.005$ vs previous observation; ${ }^{\top} P<0.000$ I vs previous observation. 


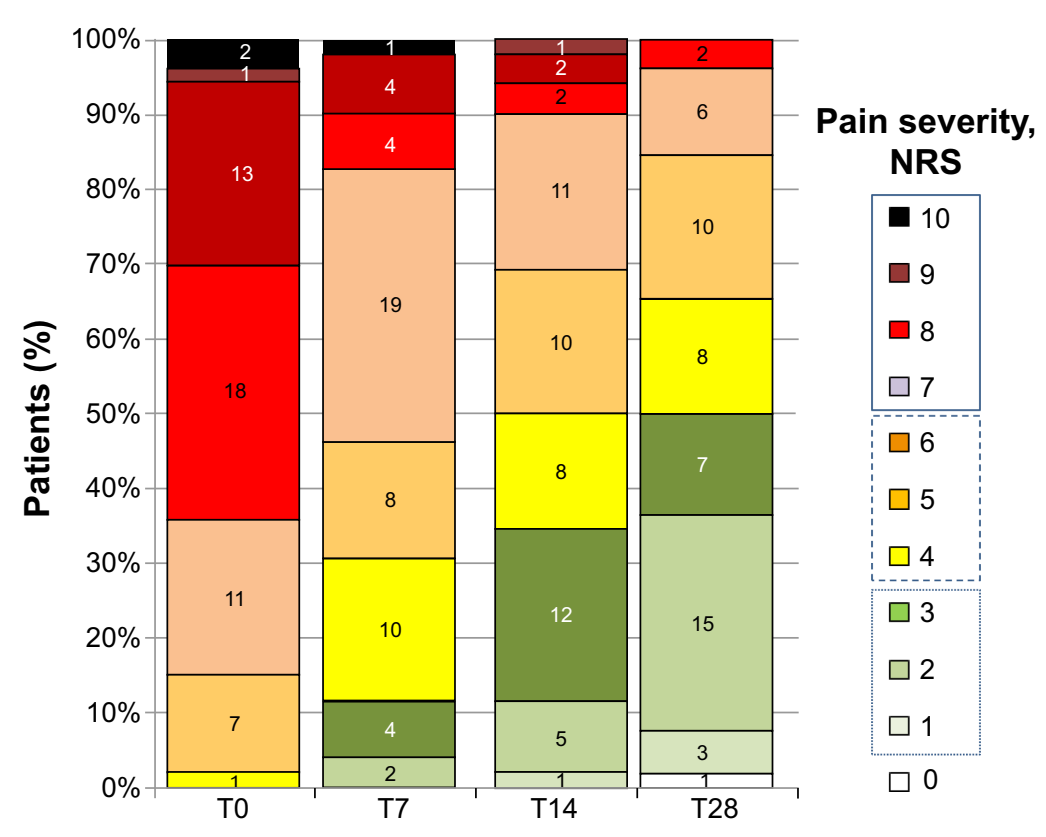

Figure 4 Proportions of patients with severe (Numeric Rating Scale [NRS] 7-10), moderate (NRS 4-6), mild (NRS I-3), or no pain (NRS 0) at baseline and throughout the observation on prolonged-release oxycodone-naloxone (OXN-PR) (baseline: $n=53$; day 7 [T7], day 14 [TI4], and day 28 [T28]: $n=52$ ). Four patients still complained of severe pain (mean NRS >6) after 28 days on OXN-PR. Three of them were on 20/10 mg OXN-PR daily and preferred not to further increase their daily dosage because of fear of increasing other symptoms potentially related to opioid (drowsiness in one, dry mouth in two). Another 85 -year-old woman with severe osteoarthritic pain reported only modest analgesic benefit at the end of the 4-week observation on OXN-PR 10/5 mg daily. OXN-PR was deliberately not increased by her physician because of her unstable gait and history of falls.

The older patients suffering chronic nononcological pain enrolled in our observational study (median age 83 years) had a significant analgesic benefit with the new OXN-PR treatment. Notably, most patients achieved these benefits with a low dose of OXN-PR ( $\leq 15 / 7.5 \mathrm{mg}$ daily) and with no need to increase to higher doses during the 28 days of observation. The low OXN-PR dosages prescribed were well tolerated, even in this population of very old patients.
Of note, even in these subjects not previously treated with a strong opioid, OXN-PR neither had a negative impact on the cognitive status nor impaired bowel dysfunction.

This is, to our knowledge, the first study specifically addressing the efficacy and tolerability of OXN-PR in older adults with chronic pain of moderate-to-severe intensity not adequately controlled by NSAIDs and/or paracetamol or WHO step II analgesics (weak opioids). The pain relief

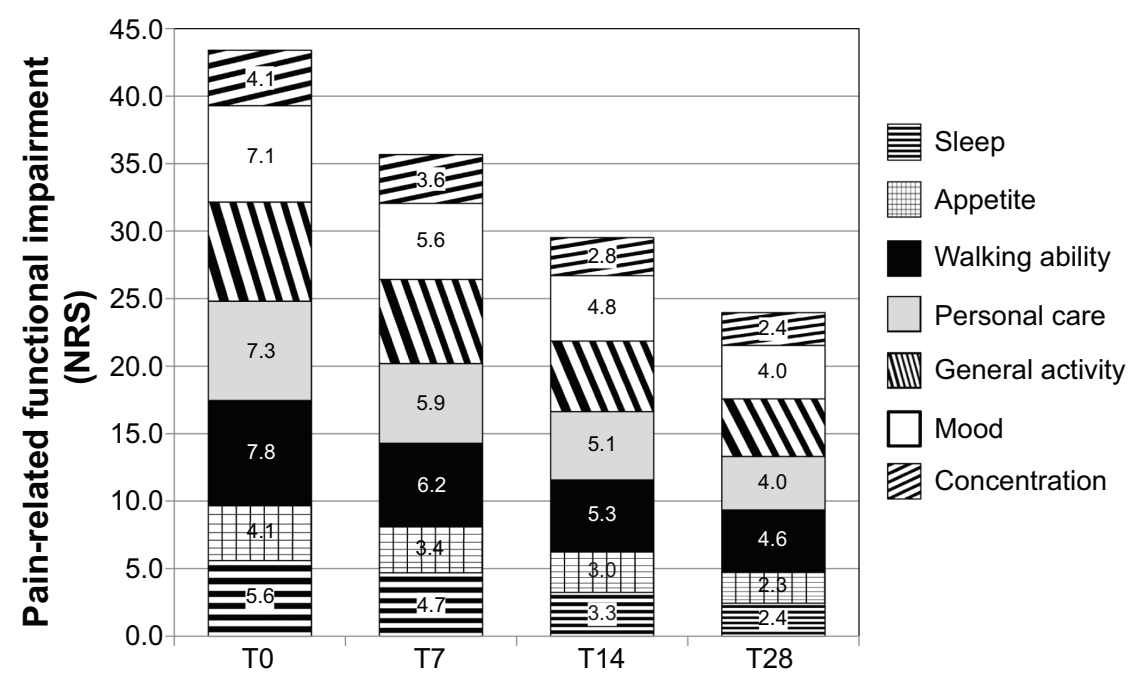

Figure 5 Mean individual domain scores for pain-related functional impairment (measured by Numeric Rating Scale [NRS]) at baseline and at day 7 (T7), day 14 (TI4), and day 28 (T28) of treatment with prolonged-release oxycodone-naloxone. 


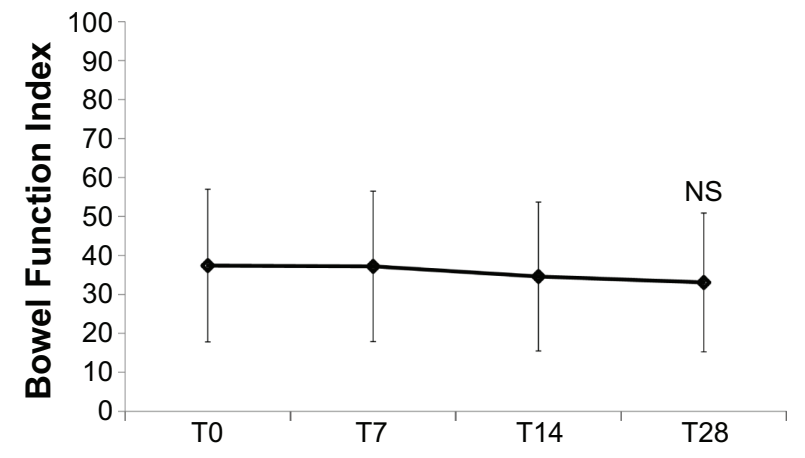

Figure 6 Constipation severity throughout the study, as assessed by Bowel Function Index (mean values \pm standard deviation).

Abbreviation: NS, variations not significantly different.

observed in our study was clinically relevant and substantial. The mean NRS score nearly halved from baseline to the end of the 28-day observation, with the 3.26 mean decrease measured on an eleven-point pain NRS being well above the two-point decrease in chronic pain intensity commonly considered as clinically meaningful. ${ }^{24}$ As we had limited data on OXN safety/tolerability and proper titration, we initiated OXN-PR cautiously and preferred to "start slow, go slow" in order to minimize potential side effects and thereby improve the compliance to opioid treatment in our fragile population. At the same time, we were cognizant of the potential for underdosing with this strategy, and thus we allowed for ample rescue pain control. In fact, no patients reported needing rescue medication more than twice in 1 day.

The present findings confirm the efficacy of OXN-PR in achieving pain relief and reducing constipation, with good tolerability suggested by subgroup analyses of larger trials and studies showing that older people can also benefit significantly from this novel combination. ${ }^{25-27}$ In a large observational study, Gatti et $\mathrm{al}^{25}$ investigated the effectiveness and tolerability of OXN-PR in different age subgroups of patients with constipation and chronic noncancer pain of moderate-to-severe intensity. Overall, age did not affect treatment outcomes and tolerability, although some benefits (ie, magnitude of pain relief, need for rescue paracetamol, onset of perceived clinical benefit, as well as improvement in constipation) were greater in the oldest subgroup. In that study, however, the effects of OXN-PR on functional and cognitive status, mood, and quality of life of older patients were not specifically addressed, and the retrospective design of their investigation could have biased some results.

The present study is the first establishing the efficacy of low-dose OXN-PR in the elderly. Our findings also add to the evidence that the use of strong opioids (step III of the WHO ladder) at low doses is a valid option for the treatment of moderate-to-severe pain not adequately controlled with paracetamol and/or NSAIDs, and that skipping step II of the WHO ladder is feasible, as suggested by current guidelines on pain management in older people. ${ }^{4}$ According to current guidelines, opioid treatment should be initiated at the lowest possible dose, slowly uptitrated, and carefully tailored based on response and tolerability of the patient. ${ }^{3,4}$ Older people usually require lower doses than younger individuals; however, the efficacy of opioids does not appear to be influenced by age. ${ }^{4,28}$

Effective analgesia with low-dose OXN-PR resulted in significant improvements in quality of life and mood disorders, and significantly increased the ability to perform activities of daily living. These findings, which clearly need to be investigated in more detail, are of potential relevance considering the close interconnection between pain, mood, sleep quality, and quality of life in the elderly., 4,29,30

Constipation is the most frequent and persistent $\mathrm{AE}$ induced by opioids, ${ }^{9}$ and, in older patients, who show a higher incidence of bowel dysfunction, opioid-induced constipation is a common complication that should be anticipated. This is reflected in current guidelines that recommend the use of laxatives or oral OXN-PR for the prevention and treatment of constipation. ${ }^{4}$ The treatment with OXN-PR did not worsen bowel dysfunction in our patients, even in those subjects with manifest constipation at baseline, with a nonsignificant decrease from baseline in the proportion of patients complaining of constipation after 28 days of treatment and without an increase in laxative consumption.

The loss of cognitive function possibly induced by long-term opioid treatment is another major concern in the management of chronic pain in the elderly. The available evidence suggests that cognitive function and behavior are not significantly affected in patients taking stable doses of opioids, while a dose increase may be associated with impaired cognitive function. ${ }^{4,31-33}$ On the other hand, it is reasonable to expect that potential negative effects in cognitive function may be outbalanced by the beneficial effects provided by pain relief on mood, ability to perform daily tasks, and quality of life. ${ }^{30}$ In our findings, treatment with low-dose OXN-PR did not impact negatively on the overall cognitive and behavioral status of the study population, which, however, did not include patients with manifest cognitive impairment. The issue regarding pain treatment in patients with cognitive impairment is of great relevance, especially given the strong correlation between cognitive impairment and inadequate pain assessment and treatment in cognitively impaired older adults. ${ }^{34,35}$ Efficacy and tolerability of the 


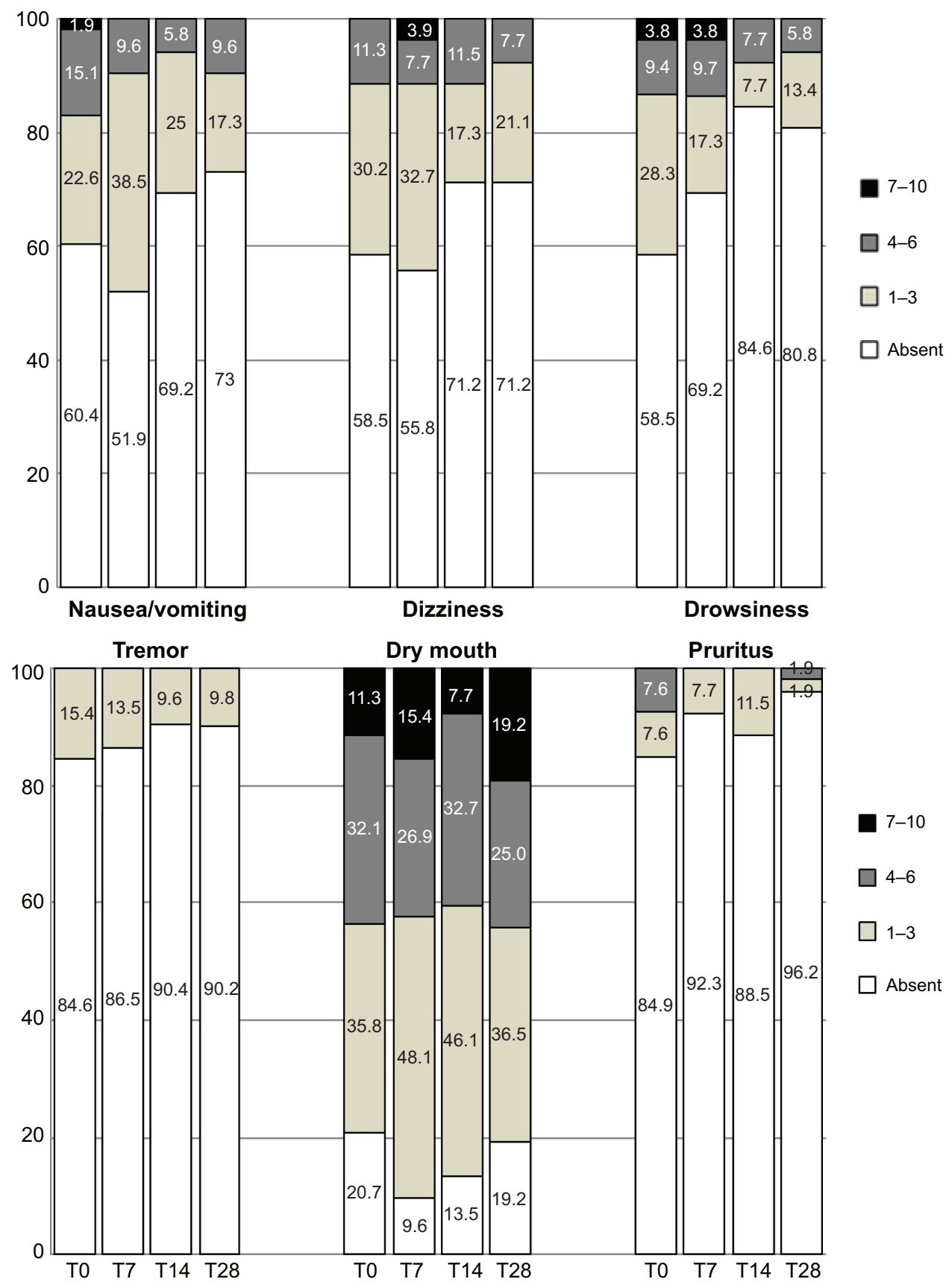

Figure 7 Proportions of patients affected by disorders commonly related to opioids and their severity at different time points of the observation. Symptom severity rated on an eleven-point scale $(0=$ absent; $1-3=$ mild; $4-6=$ moderate, $7-10=$ severe). All differences in frequency distributions were not significant (corresponding $P$-values $>0.0083$ at all times).

OXN-PR combination in the elderly with cognitive impairment deserves further ad hoc analysis.

Drowsiness and dizziness are other central opioidinduced AEs of particular concern in older patients, as they have been associated with an increased risk of falls and fractures in opioid users. ${ }^{36}$ In our prospective study, no falls were registered, and, as a matter of fact, prevalence and severity of commonly opioid-related symptoms were already present before the switch to OXN-PR, and most of them decreased in the following weeks, with the exception of dry mouth, which increased slightly over time.

Opioids may suppress saliva production, causing unpleasant dry mouth symptoms. In a systematic review of randomized trials with oral opioids in nonmalignant pain, dry mouth was reported as one of the most common AEs, affecting $25 \%$ of active treatment patients and only $3.2 \%$ of those in the placebo arm. ${ }^{37}$ It is unknown whether older patients are more affected than younger ones by this tedious 
side effect. In a pooled analysis of double-blind, randomized, controlled trials comparing oxycodone PR alone and with naloxone (OXN-PR) in patients with moderate/severe nonmalignant pain and opioid-induced bowel dysfunction, the rates of dry mouth were low (1\%) and comparable in both treatment groups; ${ }^{38}$ in the large observational study by Gatti et $\mathrm{al}^{25}$ assessing effectiveness and safety of OXN-PR in consecutive nononcological patients with chronic pain despite analgesic treatment and constipation, only one patient $(0.1 \%)$ complaining of severe dry mouth required OXN-PR discontinuation. Thus, in patients on opioids complaining of constipation, the true prevalence of other less bothersome side effects such as dry mouth could be under-reported. In our patients, presence of dry mouth was actively assessed from the beginning of our observation, and high rates of moderateto-severe dry mouth were reported both before (43.4\%) and on OXN-PR ( $44.2 \%$ after 4 weeks of active treatment).

Besides the observational design and the absence of a control group, the small number of subjects enrolled and the short treatment duration are important limitations of the present study. On the other hand, given the relatively simple inclusion criteria used (ie, patients with moderate-to-severe chronic pain naïve to strong opioids), this study describes a real-world scenario of management in a setting that, in our opinion, closely reflects the real-world management of older patients with moderate-to-severe chronic pain followed by office-based physicians.

\section{Conclusion}

The findings of this prospective study involving older patients suffering from chronic pain of moderate-to-severe intensity suggest that low doses of OXN-PR provided effective analgesia and were generally well tolerated with no occurrence of constipation and other opioid-related complications. The benefits of the treatment also included significant improvements in quality of life and functional state. While this is not a double-blind, randomized, controlled clinical trial, the data suggest that low-dose oral OXN-PR appears to be a valid treatment option for persistent moderate-to-severe pain in geriatric patients with an insufficient response to paracetamol and/or NSAIDs or weak opioids. Our findings provide useful guidance for future studies to confirm the appropriate use of OXN-PR in the management of old and very old ( $>80$ years) patients suffering from chronic pain.

\section{Acknowledgments}

Editorial assistance (English language editing and styling) was provided by Mary Hines, on behalf of HPS Publishing and
Services Srl. This assistance was funded by Mundipharma Pharmaceuticals Srl, Italia.

\section{Disclosure}

The study was completed independently with no funding. The authors report no conflicts of interest in this work.

\section{References}

1. Davis MP, Srivastava M. Demographics, assessment and management of pain in the elderly. Drugs Aging. 2003;20(1):23-57.

2. Gibson SJ, Lussier D. Prevalence and relevance of pain in older persons. Pain Med. 2012;13 Suppl 2:S23-S26.

3. Chai E, Horton JR. Managing pain in the elderly population: pearls and pitfalls. Curr Pain Headache Rep. 2010;14(6):409-417.

4. Abdulla A, Bone M, Adams N, et al. Evidence-based clinical practice guidelines on management of pain in older people. Age Ageing. 2013; 42(2):151-153.

5. Kahan M, Srivastava A, Wilson L, Mailis-Gagnon A, Midmer D. Opioids for managing chronic non-malignant pain: safe and effective prescribing. Can Fam Physician. 2006;52(9):1091-1096.

6. Trescot AM, Helm S, Hansen H, et al. Opioids in the management of chronic non-cancer pain: an update of American Society of the Interventional Pain Physicians' (ASIPP) Guidelines. Pain Physician. 2008;11(2 Suppl):S5-S62.

7. Bell TJ, Panchal SJ, Miaskowski C, Bolge SC, Milanova T, Williamson R. The prevalence, severity, and impact of opioid-induced bowel dysfunction: results of a US and European Patient Survey (PROBE 1). Pain Med. 2009;10(1):35-42.

8. Kurz A, Sessler DI. Opioid-induced bowel dysfunction: pathophysiology and potential new therapies. Drugs. 2003;63(7):649-671.

9. Pappagallo M. Incidence, prevalence, and management of opioid bowel dysfunction. Am J Surg. 2001;182(5A Suppl):11S-18S.

10. American Geriatrics Society Panel on Pharmacological Management of Persistent Pain in Older Persons. Pharmacological management of persistent pain in older persons. $J$ Am Geriatr Soc. 2009;57(8): 1331-1346.

11. Mercadante S, Giarratano A. Combined oral prolonged-release oxycodone and naloxone in chronic pain management. Expert Opin Investig Drugs. 2013;22(1):161-166.

12. Choi YS, Billings JA. Opioid antagonists: a review of their role in palliative care, focusing on use in opioid-related constipation. $J$ Pain Symptom Manage. 2002;24(1):71-90.

13. Liu M, Wittbrodt E. Low-dose oral naloxone reverses opioidinduced constipation and analgesia. J Pain Symptom Manage. 2002; 23(1):48-53.

14. Lowenstein O, Leyendecker P, Hopp M, et al. Combined prolonged-release oxycodone and naloxone improves bowel function in patients receiving opioids for moderate-to-severe non-malignant chronic pain: a randomised controlled trial. Expert Opin Pharmacother. 2009;10(4): 531-543.

15. Vondrackova D, Leyendecker P, Meissner W, et al. Analgesic efficacy and safety of oxycodone in combination with naloxone as prolonged release tablets in patients with moderate to severe chronic pain. J Pain. 2008;9(12):1144-1154.

16. Simpson K, Leyendecker P, Hopp M, et al. Fixed-ratio combination oxycodone/naloxone compared with oxycodone alone for the relief of opioid-induced constipation in moderate-to-severe noncancer pain. Curr Med Res Opin. 2008;24(12):3503-3512.

17. Magni E, Binetti G, Bianchetti A, Rozzini R, Trabucchi M. Mini-Mental State Examination: a normative study in Italian elderly population. Eur J Neurol. 1996;3(3):198-202.

18. Mahoney FI, Barthel DW. Functional evaluation: the Barthel Index. Md State Med J. 1965;14:61-65.

19. Cleeland CS, Ryan KM. Pain assessment: global use of the Brief Pain Inventory. Ann Acad Med Singapore. 1994;23(2):129-138. 
20. Bonezzi C, Nava A, Barbieri M, et al. [Validazione della versione Italiana del Brief Pain Inventory nei pazienti con dolore cronico]. Minerva Anestesiol. 2002;68(7-8):607-611.

21. Rentz AM, Yu R, Muller-Lissner S, Leyendecker P. Validation of the Bowel Function Index to detect clinically meaningful changes in opioid-induced constipation. J Med Econ. 2009;12(4):371-383.

22. Rentz AM, van Hanswijck de Jonge P, Leyendecker P, Hopp M. Observational, nonintervention, multicenter study for validation of the Bowel Function Index for constipation in European countries. Curr Med Res Opin. 2011;27(1):35-44.

23. Gianni W, Madaio RA, Di Cioccio L, et al. Prevalence of pain in elderly hospitalized patients. Arch Gerontol Geriatr. 2010;51(3):273-276.

24. Farrar JT, Young JP Jr, LaMoreaux L, Werth JL, Poole RM. Clinical importance of changes in chronic pain intensity measured on an 11-point numerical pain rating scale. Pain. 2001;94(2):149-158.

25. Gatti A, Casali M, Lazzari M, et al. Prolonged-release oxycodone/ naloxone in nonmalignant pain: single-center study in patients with constipation. Adv Ther. 2013;30(1):41-59.

26. Lux EA, Leyendecker P, Kremers W, Bosse B, Hopp M, Reimer K. Efficacy and safety of oxycodone and naloxone in a prolonged release fixed combination tablet in elderly patients ( $>65$ years) with moderate to severe chronic pain [abstract PB7 475]. Paper presented at: 19th World Congress of Gerontology and Geriatrics; Jul 5-9, 2009; Paris, France.

27. Schutter U, Grunert S, Meyer C, Schmidt T, Nolte T. Innovative pain therapy with a fixed combination of prolonged-release oxycodone/ naloxone: a large observational study under conditions of daily practice. Curr Med Res Opin. 2010;26(6):1377-1387.

28. Mercadante S, Ferrera P, Villari P, Casuccio A. Opioid escalation in patients with cancer pain: the effect of age. J Pain Symptom Manage. 2006;32(5):413-419.

29. Husebo BS, Ballard C, Sandvik R, Nilsen OB, Aarsland D. Efficacy of treating pain to reduce behavioural disturbances in residents of nursing homes with dementia: cluster randomised clinical trial. $B M J$ 2011;343:d4065.
30. Landi F, Onder G, Cesari M, Russo A, Barillaro C, Bernabei R. Pain and its relation to depressive symptoms in frail older people living in the community: an observational study. J Pain Symptom Manage. $2005 ; 29(3): 255-262$.

31. Chapman SL, Byas-Smith MG, Reed BA. Effects of intermediate- and long-term use of opioids on cognition in patients with chronic pain. Clin J Pain. 2002;18(4 Suppl):S83-S90.

32. Kamboj SK, Tookman A, Jones L, Curran HV. The effects of immediaterelease morphine on cognitive functioning in patients receiving chronic opioid therapy in palliative care. Pain. 2005;117(3):388-395.

33. Tassain V, Attal N, Fletcher D, et al. Long term effects of oral sustained release morphine on neuropsychological performance in patients with chronic non-cancer pain. Pain. 2003;104(1-2):389-400.

34. Bernabei R, Gambassi G, Lapane K, et al. Management of pain in elderly patients with cancer. SAGE Study Group. Systematic Assessment of Geriatric Drug Use via Epidemiology. JAMA. 1998;279(23): $1877-1882$.

35. de Souto Barreto P, Lapeyre-Mestre M, Vellas B, Rolland Y. Potential underuse of analgesics for recognized pain in nursing home residents with dementia: a cross-sectional study. Pain. 2013;154(11):2427-2431.

36. Vestergaard P, Rejnmark L, Mosekilde L. Fracture risk associated with the use of morphine and opiates. J Intern Med. 2006;260(1):76-87.

37. Moore RA, McQuay HJ. Prevalence of opioid adverse events in chronic non-malignant pain: systematic review of randomised trials of oral opioids. Arthritis Res Ther. 2005;7(5):R1046-R1051.

38. Lowenstein O, Leyendecker P, Lux EA, et al. Efficacy and safety of combined prolonged-release oxycodone and naloxone in the management of moderate/severe chronic non-malignant pain: results of a prospectively designed pooled analysis of two randomised, double-blind clinical trials. BMC Clin Pharmacol. 2010;10:12.
Clinical Interventions in Aging

\section{Publish your work in this journal}

Clinical Interventions in Aging is an international, peer-reviewed journal focusing on evidence-based reports on the value or lack thereof of treatments intended to prevent or delay the onset of maladaptive correlates of aging in human beings. This journal is indexed on PubMed Central, MedLine,

\section{Dovepress}

CAS, Scopus and the Elsevier Bibliographic databases. The manuscript management system is completely online and includes a very quick and fair peer-review system, which is all easy to use. Visit http://www.dovepress. com/testimonials.php to read real quotes from published authors. 